Succinin nennt der Verf, eine Substanz, die durchs Erbiteen ron bernsteinsaurem Kalk erhalten werden kann, und viele Aehnlickeit mit andern brenzlichen Körpern hat.

(Aneales de chim T. LXI.)

VIERTER ABSCHNLTT.

Organische Bildungstheile von Pflanzen und deren Producte.

\title{
Ueber die Stărke;
}

Bericht der Akademie der Wissenschaften zu Paris über mehrere Abhandlungen der Herm Payen und Persoz, Couverchel, Guérin-Varry und Lassaigne, abgestattet von

\section{Dulong, Dumas, Robiquet und Chevreul.}

In den letzt verflossenen Jahren sind viele Arbeiten über die Stärue von mehreren jungen Chemilern der Akademie vorgelegt worden. Hierbei geschah es auch, wie immer, wenn mehrere Beobachter sich mit demselben Gegenstande beschäf. tigen, dafs die Beobathtungen nicht mit einander äbereinstimmten, und dals mehrere sich um die Ehre der ersten Entdeckung stritten. Es hat daher die Akadenie eine Commission zur Prüfung der ihr vorgelegten Arbeiten niedergesetzt.

Der Bericht dieser Commission zertällt in $2 \mathrm{Abschnitte;}$ der erste enchält eine Uebersicht aller ältern Arbeiten über das Stärkeraehl, der $\mathrm{z}$ weite bezieht sich anf die, weiche der A thadernie neuerlich vorgelegt worden sind.

Den Inhalt des ersten Abschnilts dieses Berichtes dürfen wir als wohl beliannt hier voraussetzen, ond uns daher nur 
bei einer Bemerkng über die Arbeit ron Biot und Persos anflalten. Dieselben haben, sagen die Berichterstatter, in ihren Untersuchungen über die Stärke eine ron Biot entdeckte optische Eigenschaft zu benutzen gesucht. Sie batten die Beobachtung gemacht, dafs Anfösungen von gewissen Planzenstoffen auf einen durch sie hindurchgehenden polariairten Lichtstrabl eine solche Wirkung ansüben, dafs die Richtang der Polarisationsebene geindert erscheint, and dafs man diese Aichtungsveränderung genau messen bồnne. Biot meinte dann, dafs man diese, ron der Lage der Atome abhängige, Eigenschaft benutzen konnne, um nicht allein K8rper, welche sio beaitzen, ron ondern, die sie nicht besitzen, su unterscbeiden, sondern such, um Körper von eüander za nnterscheiden, welche sie in verschiedenen Greden zeigen.

Es ist aber sehr die Frage, ob es hinreichend ist, eine physische Eigenschat eines Korpers erhannt za haben, and die verschiedenen Grade ihrer Intensität messen zu ionnnen, um sie anch als ein characteristisches Merkmal für die Beatimmang der chemischen Natar eines Hörpers anwenden zu bŏn. nen. Wir zweifeln sehr daran. Um hierübor Gewifsheit zo erlangen, mafs man diese Eigenschaft bei einer Reibe roa möglichst homogenen liörpern stadiren, and betimmen, in welchem Grade jeder von ibnen sie auf eine constante Art bei bostimnten Temperatur., Anflösungs - und andern Yerbältnisseu zeigt. In der That, wenn Temperalarveranderungen, Veränderangen in der Aullöslichkeit des Hörpers; mit eịnem Worte, Yerhältnisse welche für den Chemiker zu gerieg sind, weil die Hauplcharahtere der Art dus Körpers nicht durch sie modificirt worden, irgend eine Wirkung auf die Intensität der besagten Eigenschaften susüben, so kann der Chomiher diese Eigenschaft, obgleich sie für den Physiker von gros. sem Interesse ist, dennoch nicht als ein Hauptmerkmal betrachten. Es wäre dies eben so der Fall, and noob mehr, 
wenn diese Eigenschaft aus der Vereinigung von wenig verwandten Körpern entsprängen, wio z. B. von Körpern, deren Verwandtschaft so schwach ist, wie die eines neutralon $\Delta$ uflöonngsmittels za einem ebenfalls neatralen löslichen Körper*).

In dem zweiten Abschnitte liefert $\mathrm{Che}$ rreal die folgende Kritil über die Abbandlangen von Paye $\mathbf{n}$ und $\mathbf{P}$ ersoz, Couvercbel, Gućrin-Varry und Lassaigac.

Die Btïrke besteht aus einer Hülle und einer innern Sabstane, welche sich sehr von einander unterscheiden, da letztere allein in heifsem Wasser aufloslich ist. Diese Thatsache wurde von Raspail anfgestellt. Es ist diese lösliche Substanz weder nach $\mathbf{R a s p a i l}$ arabisches Gummi, noch nach Biot und Persoz reines Dextrin. Denn im ersten Falle müfste die Stärke dorch Behandeln mit Salpetersäure, Schleinscuure geben, und in beiden müfste haltes Wasser fein zertheilte Stärbe leicht auflōsen, wie diefs beim arabischen Gum. mi und dem Dextrin der Fall ist. Wir müssen daher der Meinung ron Payen und Persoz and der ron Guérin eine besondere Aufmerhsamkeit schenken, da sie beine solche Schwierigkeiten darbieten. Die 20 beantwortenden Fragen sind folgende: Ist die auflösliche Substanz des Stärtmehls ein reiner, unmittelbarer Bestandtheil, die Amidone nach Payen und Persoz? oder besteht sie aus Amidin und Amidine **), wie Gućrin behauptet? Wäre sie ein einziger näherer Bestandtheil, so mülste dieser durch die Einwirkang des Hockenden Wassers genau sich in Amidinc und lösliches Amidin verwandela.

Untersuchen wir nun die Gründe, aus welchen man, der Ansicht von Payen und Persoz zufolge, die innere Substonz der Stärke, als Amidone zu betrachteu hat.

$)$ S. diese Annalen. Bd. VI. S. 200.

*) S. diese Annalen. Bd. XIII. S. 7 . 
Da die Amidone in Wasser unter $65^{\circ}$ sich nicht löst, und nur in heifsem Wasser es thut, so sieht man sogleich, warum die Stärke von kaltem Wasser so wenig angegriffen wird, and sich in Hleister verwandelt, wenn sie in einer gehörigen Quantität Wasser erhitzt wird, und in Gallerte, wenn man, nach ihrem Auflösen in Wasser, die Flüssigkeit gehö. rig eindampft und erlialten läfst.

Die Amidone ist die einzige betannte Substanz, welche durch Jod blau wird; sie fällt ferner Barytwasser, basischeseigsaures Blei und Galläpfelinfusion. Darans schliefsen Payen and Persoz, dafs, wenn diese Eigenschaften sich bei Substanzen zeigen, welche aus Stärke erhalten wurden, die der Einwirkung der Hitze, des warmen Wassers, der Diastase, der Säuren n. s. w. ausgesetzt wurden, nothwendig ein Antheil der Amidone der Stärke dem einwirkenden - Kồrper widerstanden haben müsse. So $2 . \mathrm{B}$. behaupten sie, dafs die Eigensch\&ft der Amidine, des löslichen Amidins und des Hüllenamidins von Guérin durch Jod gefärbt zu werden, von der Amidine abhängig sey.

Dieser Schlufs erscheint sebr befriedigend; denn es ist natürlicher, blos einem einzigen Kōrper eine merkwürdige Eigenschaft, welche als Bezeichnung eines Charabter dienen Lann, zuzuschreiben, als mehreren Horpern. Andrerseits aber, wenn mebrere Substanzen aus einem einzigen Ganzen eine solche Eigenschaft zeigen, so kann man doch nicht immer daraus schliefsen, dafs diese Substanzen nur unreine Pro. ducte seyen. Wir meinen, dafs ein Chemiker durch eine solche Erscheinung sich immer angespornt fühlen muft, be sondere Versuche anzustellen, um $\mathrm{zn}$ untersuchen, ob diese den Substanzen gemeinschaftliche Eigenschaften blos einem einzigen Hörper angebören. Nur dann lärst sich ein genügender Schlufs darans ziehen wenn diese Versuche, unter 
Vergleichung der Resaltate und der Araft der angewendeten Agentien angestellt worden sind.

Weil die Stärke darch die Einwirkng der Schwefelsănure sich gänzlich in featen Zacker verwandelt, will man behaupten, dafs defshalb nur ein einziges Princip, nämlich die Amidone in der Stärke rorhanden seyn könne. Diese Behauptung verliert aber ihren Werth, wenn man bedentst, daf sebr viele, sebr ron einander verschiedene, unmittelbare Bestandtheile, gleich der Stärke, diese Umwandlung erleiden. Es wäre also möglich, dafs sich in der Stärke zwei Principien, die Amidine und das lösliche Amidin befänden, welche diese Figenschaft zeigten. Denn jedesmal, wenn die Einwirhung der Schwefelsäure nicht vollständig erfolgt, bildet sich eine unkrystallisirbare, geschmacklose nnd nicht gährungsfäbige Substanz. Aus dieser Erscbeinung liefse sich dann wiederum auf dio rorige Hypothese entgegnen, dafs eine der vorhandenen Principien mehr als das andere geneigt sey, sich in Zucker zu verwandeln. Diese Annabme wäre eben so wahrscheinlich, als die vorige, wo man annimmt, dufs ein einziges Princip dor Stärbe, ebe es ganz zu Zucker wird, sich in eine nicht zuckerartige, auflösliche Substanz verwandelt.

Wir wollen nun auch die Gründe untersuchen, welche Guéri n bewogen, die Stärke als ans Amidine, löalichem Amidin und Hüllen. Amidin zusammengesetzt za betrachten.

Nach Guerin ist die innere Substanz der Stärbe eine Verbindung ron Amidine und löslichem Amidin. Briagt man sie mit siedendem Wasser in Berübrung, so löst sie sich gant darin auf, mit Ausnohme tines Theils, welcher mit der Hälle zurückbleibt, und das Hüllenamidin (tegumentüre Amidin) bildet. Concentrirt man die filtrirte Flüssigbeit, und läfst sie sorgfaltig erkalten, so subeidet sich das löslicbe Ansidin als gallertartige Masse aus. Man kann selbst eine ordentliche Gallerte erhalten, weiche nichts anderes ist, als ein yon dea Te- 
gumonten befreiter Stärkelileister. Dampft man sie zur TrocLenbeit $a b$, und nimmt den Büclstand wieder mit Wasser ouf, so lost sich die Amidine mit etwas Amidin auf, und der Rückstand ron Amidin enthëlt etwas Amidinc. Man erulärt diefs dorch die Unlöslichteit des Amidins und die Verwandischaft der Amidine zum Wasser.

Die Gründe, welche Guérin bewogen, die Amidine und das lösliche Amidin als unmittelbare Principien der Stärke anzunehmen, sind folgende: Die Amidine ist neutral and farblos wie die Stïrke; es wird stark durch Jod gefarbt; das Wasser zieht Amidine and I6sliches Amidin aus der Stärke aus. Was den letzteren Umstand betriflt, so machto Guérin folgenden Versuch in Gegenwart der Commission: Ein Papierfilter warde in einen unten zageschmolzenen Trichter gesetzt; dann wurde Wasser darauf gegossen, bis der Trichter und das Papierfilter halb voll waren. Dann brachte man auf das Papier zerriebeno. Stärte und so viel Wasser, um des Filtrum ganz za näsuen. Endlich brachte man, mittelat einer sugezogenen Pipette, eine Schicht Jodwasser auf den Boden des Trichters. Inverhalb einiger Minuten bemerlate man gar leine Färbung, was bewies, dafe beine Stärke durch das Filter gegangen war. Endlich aber, nach 6 bis 12 Minuten, sah man farblose Streifen, welche sich sogleich blau färbten, so wie sie mit dem Jod zusammenbamen. Diese Erscheinung hann aber blos ron einem anfgeIósten Körper herrübren. Ans diesem Versuche scheint also hervorzngehen, dafs die Stärke an das kalte Wasser einé auflösliche Substanz abtritt, welehe, in Berübreng mit Jod, blaa wird, and defs diese Erscheinang nicht ron einem Antheile ron in Wasser suspendirter Stärke herrührt. Kann man aber daraas, dafs eine in Wasser Iosliche, darch Jod blan werdende Substanz, durch das kalte Wasser der Stärke entzogen wird, schliefsen, dafs die ganze, in heifsem Wasser 
lösliche, Stärbe nur ans Amidine und Iöslichem Amidin bestehe? unả dafs ferner dicse beiden Substanzen sich schon vorher in der Stärke befänden, ehe sie mit heifsem Wasser behandelt wurde? Aus folgenden Gründen bönnen wir dieser Bebarptung nicht beistimmen:

1) Es könnte der Fall seyn, dafs eine Substanz, ron der Art wie die Amidine, das Vermōgen besäfse, sich darch Jod zu bläuen, wie die Amidine und das Amidin, in welche sie sich durch die Einwirkung des Wassers und einer gehzrigen 'Temperatur verwandelte; alsdann hönnte man ans der Färbung des Jods durch Stärke nicht mit mehr Recht anf die Gegenwart der Amidine and des Amidins in der Stärke schliefsen, als ans der Färbung der Auflösung mit kaltem Wasser auf die der Amidone.

2) Die Substanz, welche sich durcb Jod blau färbt, und aus der zerriebenen Stärke durch baltes Wasser ausgezogen wird, bann, in Bezug auf die unlösliche Masse, in so geringer Quantität vorhanden seyn, dafs man nicht, wie Guérin es that, schon schliefsen kann, dafs die Stärke ungeföhr 0,60 Amidine enthalte. Angenommen, das kalte Wasser löse sur sebr wenig von jer Stärke auf, das heifse hingegen löse 0,60 'Ibl. ron in kaltem Wasser löslicher Amidine auf, nachdem das Amidin davon entfernt wurde, so scheint es natūrlicher, dafs eine Substanz, wic die Amidone, die ganze Stärke ansmache, und dafs letztere nur durch die Einwirlung ron heifsem Wasser in Amidine and Amidin rerwandelt werde.

Was das Hüllen - Amidin betrifft, so besteht es wahr. cheinlich aus Tegument und derselben Substanz, welehe die Hauptmasse des löslichen Amidins ausmacht, Es scheint übrigens das Tegument, wenn nicht der llolzfaser ganz gleich, doch wenigstens ihr sehr ähalich 20 seyn. Das einfachste Verfahren, es rein darzustellen, scheint das ron $\mathbf{P}$ ay e $\mathrm{n}$ 
und Persoz zu reyn; es besteht darin, dafs man die Stärke mit einer hinlünglichen Menge ron Diastase bebandelt. Wenn wir aber annehmen, dafs durch die Diastase das Tegument der Stärbe ron der inneren Substanz isolirt werde, dürfen wir, wie Payen und Persoz, daraus schliefsen, dafs die Eigenschaften der Amidine and löslichen Amidins sich in Berührang mit Jod blac zu förben, von der Amidone oder damit gemengter unverwandelter Stärke herrübre? Gewif's aicht, denn wir müfsten dann auch als bewiesen annehmen, dafs es nur eine einzige Substanz, nämlich die Amidone, gebe, welche durch Jod blau gefïrbt wird, was uns nicht so ausgemacht zu seyn scheint, wie Payen nad Pe rsoz glauben.

Ans allem diesen ergiebt sich folgendes:

1) Da die Analyse der Stärke ron Guéri n mit beifsem Wasser rorgenommen wurde, so kann man, nach den Modificationen, welche viele organische Substanzen durch das Wasser and die Hitze erleiden, annehmen, dafs die Amidine und das lösliche Amidin nur das Resultat einer unter solchen Umständen statt gefundenen Umwandlung sind. Man toŏnnte noch folgenden Versuch anstellen: ein bestimmtes Gewicht gepulverter Stärke rolbtündig mit Laltem Wasser ausziehen, und die Aufösung im luftleeren Faume verdunsten lassen, die rüchständige Masse abwiegen und mit derjenigen vergtei. ehen, welche das heifse Wasser auszieht. Im Falle der erste Rüchstand beträchtlicher wäre, als der $\approx$ weite, müsse man ihn ebenfalls mit heifsem Wasser ausziehen, and sehen, wie er sich gegen die Auflösung in Haltem Wasser verbält. Wärea dann die Producte, welche das kalte Wasser und das beifse Wasser geben, qualitatir und quantitativ gleich, so konnte man mit grofser Wahrscheinlichkeit annehmen, dafs. die Amidine und das Amidin die wirklichen unmittelbaren Bestandtheile der Stärtue seyen.

2) Unsere Kenntnisse über alle Verhilinisse, welche das 
Jod, sowobl mit Stärhe, als mit den Producten, welche ans ihr, durch Behandlung mit Wasser, Säuren u. 8. w. erbelten werden, zeigt, sind zu gering and za unbestimmt, als dafs wir aus der Färbung der Amidine und des Amidins auf dio Präexistenz dieser Substanzen in der Stürlse, oder ihrer Mengung mit unverwandelter Stärke, oder mit Amidone, schliessen könnter. Unter andern müfste man folgendt Untersuchun. gen anstellen: wird die Stïrke in vollhounmen luftreiem Was. ser durch Jod blan gefärbt? Erhält maa, durch Erhiteen von Stärite in reinem oder salzigem Wasser, in verseblossenen Destillingefafsen Producte, welche durch Jod blan gefärbt werden? Erhält man durch Behandıng von Stärhe mit Diastase, in einem lufthaitigen oder lufiecren Gefisfse, ein solches Product?

Endlich wären noch zwei Reihen son Versuchen anzu= stellen. Zuerst müfste man alle die unLrystallisirbaren, geschmacllosen, in Wasser löslichen Substanzen, denen man mit Unrecbt den Namen Gummi beigelegt hat, mit einander su vergleichen suchen, z. B. diejerigen, welche durch Einwirkuug der Hitze, der Diastase, der Säuren aut die Stärke entstehen. Zweitens müfste man antersackes, ob die Amidine und das Amidin, genau unter denselben Umständen, wie oben, dieselben Producte, wie die Stärke giebt, ob diese Producte sich eben so, wie die Stärbe, verbalten, und endlich, ob beide Substanzen verschiedene odcr identische Prodacte liefern.

(Journ. f. p. Chemie, Bd. II. S. 382.)

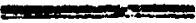

